\title{
Numerical Experiments on Anomalies from Stationary, Slowly Moving, and Fast Moving Shocks
}

\author{
Keiichi Kitamura* \\ Yokohama National University, Yokohama, 240-8501, Japan \\ and \\ Eiji Shima $^{\dagger}$ \\ Japan Aerospace Exploration Agency (JAXA), Sagamihara, Kanagawa, 252-5210, Japan
}

\section{Nomenclature}

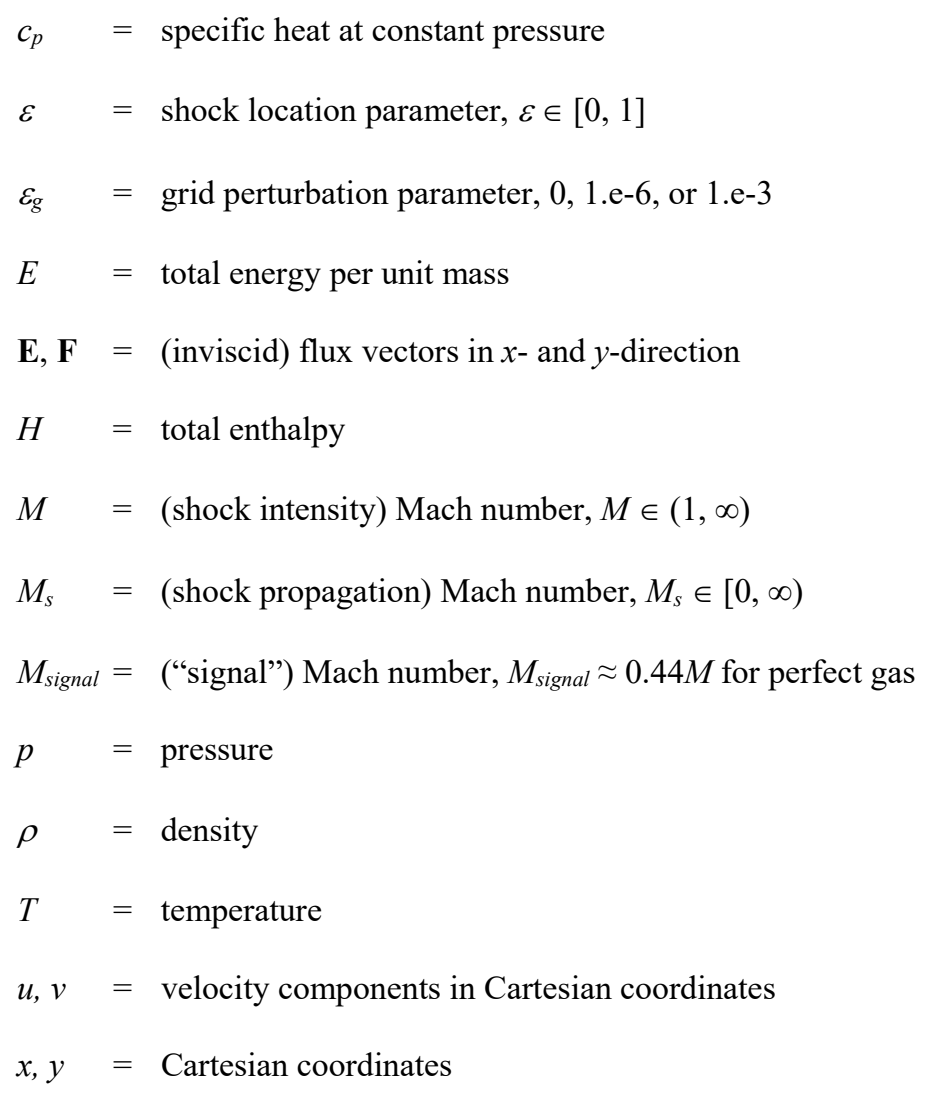

*Associate Professor, School of Engineering, 79-5 Tokiwadai, Hodogaya-ku, Senior Member AIAA.

† Senior Researcher and Director, JAXA’s Engineering Digital Innovation (JEDI) Center, 3-1-1 Yoshinodai, Chuo, Senior Member AIAA. 


\section{Subscripts}

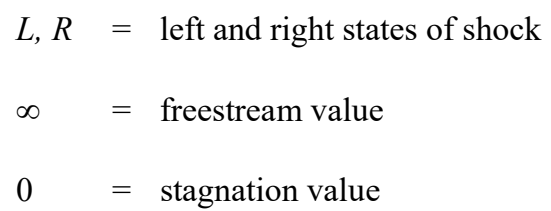

\section{I.Introduction}

Y YPERSONIC flow computations still suffer from shockwave anomalous solutions such as a "carbuncle phenomenon" (Fig. 1) [1-4]. They can severely degrade predictability of hypersonic heating, which can be disastrous to space vehicles at reentry [33]. Let us briefly look back how we have derived numerical expressions for the shockwaves (Fig. 2): i) In the real world, the shock is very thin but has a certain thickness (physically) (Fig. 2a); ii) If we express it by Euler equations (mathematically), on the other hand, we regard the shock as a discontinuous jump with zero thickness (Fig. 2b); iii) However, when the shock is captured by a finite volume method (FVM) (numerically), it should have a certain thickness of the order of the cell size (Fig. 2c). It should be cautioned that this shock thickness, along with its internal structure, is a numerical artifact, not the physical one. Within the numerical shock, the continuity equation deviates from the Hugoniot curve up to $40 \%$ at Mach 10, for instance [5]. Therefore, we take the viewpoint that the shock anomalies are caused in part by the lack of mathematical expression for internal shock structure [5-7], and hence, we think that they should be examined by numerical experiments rather than (invalid) mathematical considerations.

Quirk [1] introduced a benchmark test for numerical schemes on their responses to the captured shock waves. His test employed a particular speed of a (fast) moving shock on a two-dimensional, perturbed grid. In his test, the shock took all the possible locations within a cell but instantly passed through them. Roberts [8], on the other hand, chose a more slowly moving shock which took 50 time steps to travel through a single cell when Courant-Friedrichs-Lewy (CFL) number is unity, and discussed a post-shock numerical noise propagation. Refs. [9-12] also investigated moving shocks and post-shock oscillations both mathematically and numerically, typically in one dimension.

\footnotetext{
¥ The terms "slowly" and "fast" moving shocks, to be redefined later here, were used differently in literature: The slowly moving shock meant that the shock travels a cell with huge time in [8], [10], whereas it stood for across which shock characteristics change their signs in [11], [12].
} 
Kitamura et al. [3], inspired by [1, 5, 13,14], dealt with a stationary shock located within a row of cells in a twodimensional (unperturbed) grid, with an initial shock position parameter $\varepsilon=0.0,0.1, \ldots 0.9$, i.e., the shock was placed at one of 10 possible locations in a cell. As stated in [12] there must be at least one cell to express a shock unless it is stationary and precisely located at the cell interface $(\varepsilon=0.0)$. Thus, this set of shock locations represents every instant in which a moving shock experiences. The study in [3] discovered that one flux function has its own favorite shock location $\varepsilon$, and that such $\varepsilon$ differs from one flux to another (Fig. 3). They also revealed in Refs. [3, 15] that any flux functions including flux-vector-splitting (FVS) by Van Leer [16] can generate carbuncles, although some of those methods had been believed to be carbuncle-free according to the Quirk's test and others. In other words, Quirk's test for a moving shock does not support the robustness of a numerical flux for a stationary shock, in a strict sense. Therefore, a broader map which contains both [1] and [3] is desired as a systematic and thorough benchmark.

The present study will propose such a test by filling the gaps between the works explained above [1,3], i.e., by varying the shock propagation speed from 0 (of Kitamura et al. [3]) to 6 (in Quirk's choice [1]) and beyond, as the first attempt. Then, the effects of both shock intensity and shock propagation speed will be discussed. Following our earlier work, eight different flux functions such as by Roe [17], Van Leer [16], Liou (Advection-Upstream-SplittingMethod (AUSM)+-up [18]), and Shima and Kitamura [Simple Low-dissipation AUSM (SLAU) [19]] will be used since they have different degrees of robustness against the shock [15].

This Note is composed as follows: Sec. II will briefly explain the governing equations and numerical methods; Sec. III will present the numerical setup which also states similarities and differences between [1] and [3]; Sec. IV will show numerical results, and Sec. V will conclude the current work.

\section{II.Numerical Methods}

\section{A. Governing Equations}

The two-dimensional Euler equations are solved in this study.

$$
\begin{aligned}
& \frac{\partial \mathbf{Q}}{\partial t}+\frac{\partial \mathbf{E}}{\partial x}+\frac{\partial \mathbf{F}}{\partial y}=0 \\
& \mathbf{Q}=\left[\begin{array}{c}
\rho \\
\rho u \\
\rho v \\
\rho E
\end{array}\right], \quad \mathbf{E}=\left[\begin{array}{c}
\rho u \\
\rho u^{2}+p \\
\rho u v \\
\rho u H
\end{array}\right], \quad \mathbf{F}=\left[\begin{array}{c}
\rho v \\
\rho v u \\
\rho v^{2}+p \\
\rho v H
\end{array}\right]
\end{aligned}
$$


where $\rho$ is density, $u, v$ are velocity components in Cartesian coordinates, $E$ total energy per unit mass, $p$ pressure, $H$ total enthalpy $(H=E+(p / \rho))$, and $T$ temperature. The working gas is assumed to be air approximated by the calorically perfect gas model with the specific heat ratio $\gamma=1.4$.

\section{B. Computational Method}

The equations are discretized by a cell-centered FVM with first order both in space and time. We avoided using second or higher-order schemes, since they reportedly tend to exhibit post-shock oscillations from a moving shock more likely [8], whereas less carbuncles are produced from a stationary shock [3] - all these will possibly make our discussions more complicated. In addition, a numerical robustness of a second-order scheme against shock anomalies varied depending on a selected slope limiter in [20].

Numerical flux functions employed here are listed as follows.

1. Roe with entropy-fix (E-fix)

In terms of the robustness against the shock, this flux function is vulnerable to shock anomalies and represents Godunov [22] or Harten-Lax-van_Leer with Contact (HLLC) [23] Riemann solver according to [3, 15].

\section{Van Leer's Flux-Vector-Splitting}

This flux function is very robust against the shock, as well as Hänel [24], Harten-Lax-van_Leer-Einfeldt (HLLE) [25], SLAU2 [26], although they (except for SLAU2) are too diffusive at contact discontinuity or boundary-layer. It is modified later by Liou [27] to AUSM-family.

3. AUSM $^{+}$-up

Liou developed the AUSM-family numerical fluxes. The AUSM+-up [18], improved further from AUSM+, is employed here with tunable parameters $K_{u}=0.75, K_{p}=0.25, \sigma=1.0$ as usual. In this paper, $f_{a}$ is taken as unity, simply because only hypersonic flows are treated (please see [18] for details).

\section{SLAU}

SLAU [19] was developed by Shima and Kitamura, which is one of AUSM-family schemes for all speeds. This flux is as moderately robust against the shock as $\mathrm{AUSM}^{+}$-up.

\section{LDFSS}

LDFSS [28] was proposed by Edwards, which is also one of AUSM-family schemes. It is also as robust as AUSM+up. 


\section{6. $A U S M P W+$}

AUSMPW + [29] was developed by Kim et al., from AUSM+. With the help of its multi-dimensional dissipation term, it is very robust as Van Leer's FVS.

\section{Roe $M 2$}

Kim et al. combined Roe and HLLE fluxes into Roe M2 [30]. This is again as robust as Van Leer's FVS thanks to its multi-dimensional term.

\section{SLAU2}

Kitamura and Shima enhanced SLAU in its robustness against shock anomalies in SLAU2 [26]. This is also very robust, without a multi-dimensional dissipation though.

After the numerical fluxes through all the cell-interfaces are obtained, those contributions are integrated from cell to cell in the time evolution process, using first-order Euler explicit method. Detailed information of the solver with regard to formulations and discretizations is found in $[3,31]$.

\section{Numerical Experiments Setup}

\section{A. Basic Pre- and Post-shock Relations}

Let us consider a 1D inviscid flow setup. The flow across a normal shock wave experiences adiabatic changes in which the basic equations called Rankine-Hugoniot relations are valid as follows [32] (Figs. 4a,b):

$$
\begin{aligned}
& \frac{p_{L}}{p_{R}}=1+\frac{2 \gamma}{\gamma+1}\left(M^{2}-1\right) \\
& \frac{\rho_{L}}{\rho_{R}}=\left[1+\frac{\gamma+1}{\gamma-1}\left(\frac{p_{L}}{p_{R}}\right)\right] /\left[\frac{\gamma+1}{\gamma-1}+\left(\frac{p_{L}}{p_{R}}\right)\right]
\end{aligned}
$$

where $R$ and $L$ are pre- (upstream) and post-shock (downstream) states, respectively, in the shock coordinate system (Fig. 4b). $M$ corresponds to upstream Mach number for a stationary shock, or relative upstream Mach number for a moving shock in the shock coordinate, which is regarded as shock "intensity" Mach number, $M=\left(u_{s}-u_{R}\right) / a_{R}($ Fig.4b). Then, velocity normal to the shock is related by the continuity equation as

$$
u_{L}=u_{s}\left[1-\frac{\rho_{R}}{\rho_{L}}\right]+\frac{\rho_{R} u_{R}}{\rho_{L}} \quad\left(\because \frac{u_{s}-u_{L}}{u_{s}-u_{R}}=\left(\frac{\rho_{R}}{\rho_{L}}\right)^{-1}\right)
$$

where $u_{s}$ is shock propagation velocity and expressed by upstream condition as 


$$
u_{s}=M_{s} a_{R}, \quad a_{R}=\sqrt{2 p_{R} / \rho_{R}}
$$

where $M_{s}$ is the (moving) shock Mach number ${ }^{\S}$ [20]; apparently, $u_{\mathrm{s}}=0$ for a stationary shock.

\section{B. Post-shock "Signal" Mach Number}

Here let us consider the relation between the wave propagation speed $a_{L}$ of downstream the shock (Fig.5) and the $a_{R}$ of upstream sound speed. After some manipulation, the $a_{L}$ is written as follows:

$$
\begin{gathered}
a_{L}=\sqrt{p_{L} / \rho_{L}}=a_{R} \cdot \sqrt{\left(\frac{p_{L}}{p_{R}}\right) /\left(\frac{\rho_{L}}{\rho_{R}}\right)}=a_{R} \cdot M_{\text {signal }} \\
M_{\text {signal }}(M) \equiv \sqrt{\frac{1+\frac{2 \gamma}{\gamma+1}\left(M^{2}-1\right)}{\left[1+\frac{\gamma+1}{\gamma-1}\left(1+\frac{2 \gamma}{\gamma+1}\left(M^{2}-1\right)\right)\right] /\left[\frac{\gamma+1}{\gamma-1}+\left(1+\frac{2 \gamma}{\gamma+1}\left(M^{2}-1\right)\right)\right]}} \\
\stackrel{M \rightarrow \infty}{\frac{\sqrt{2 \gamma(\gamma-1)} M}{\gamma+1}} \approx 0.44 M \quad(\text { if } \gamma=1.4)
\end{gathered}
$$

where $M_{\text {signal }}$ is defined as a "signal" Mach number, which is a function of only one parameter, i.e., the shock intensity $M$. It is interesting to observe that $M_{\text {signal }}$ asymptotes to $0.44 M$ for $M>>1$ (Fig.6) ${ }^{* *}$. Then, we can compare $M_{s}$ with $M_{\text {signal }}$ (or $0.44 M$, instead): if $M_{\text {signal }}>M_{s}$, numerical noises in the left (downstream) region catches up with the moving shock and can disturb it; on the other hand, if $M_{\text {signal }}<<M_{s}$, the shock moves fast enough to escape from the influences of the left region (Fig.5).

We have neglected the convection speed $u_{L}$ of the left region only for brevity, but the actual maximum information propagation speed is $\left(a_{L}+\left|u_{L}\right|\right)>a_{L} \approx 0.44 M a_{R}$. Nevertheless, $M_{\text {signal }}$ serves as a good approximation for the lower bound of $\left(a_{L}+\left|u_{L}\right|\right)$. We will come back later to this important number.

\section{Stationary Shock Setup}

If a normal shock is placed at a fixed location, the equations (7)-(9) become simpler since $M_{s}=u_{s}=0$. Kitamura et al. [3] varied $M$ from 1.5 to 20 for both $1 \mathrm{D}$ and 2D stationary shocks, and discovered that the higher the $M$ was, the more likely the shock anomalies emerged, and that such a sensitivity on $M$ died out for $M \geq 6\left(M_{s}=0\right)$. At $M=6$, for instance, the pre- and post-shock states following the Rankine-Hugoniot relation (Eq. (7)) are given as:

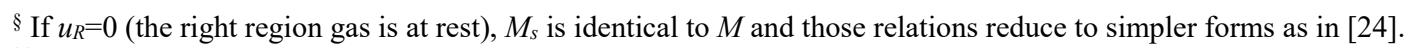

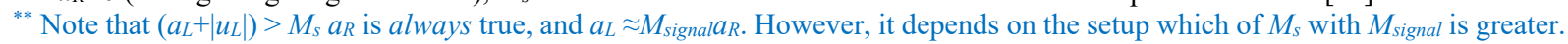




$$
\begin{aligned}
\left(\rho_{L}, u_{L}, v_{L}, p_{L}\right) & =(5.2683,1.1389,0,29.881): \text { Post-shock } \\
& =\left(5.2683,0.4042 a_{L}, 0,41.83 p_{R}\right) \\
\left(\rho_{R}, u_{R}, v_{R}, p_{R}\right) & =(1,6,0,1 / \gamma): \text { Pre-shock }
\end{aligned}
$$

The other parameters involved in [3] were shock initial locations $\varepsilon$, flux functions, and spatial accuracy. The shock initial locations included several possible positions "inside" cells. In this study, however, the shock initial location will be fixed precisely on the cell interface $(\varepsilon=0.0)$. Thus, the stationary shock $\left(M_{s}=0\right)$ is merely a special case of the moving shocks in which the shock takes the only one prescribed location relative to the grid line.

\section{Moving Shock Setup by Quirk [1]}

Quirk [1] proposed a moving shock test with $M=M_{s}=6\left(u_{R}=0\right)$ :

$$
\begin{aligned}
& \left(\rho_{L}, u_{L}, v_{L}, p_{L}\right)=(5.2683,4.8611,0,29.881): \text { Post-shock; } \\
& \left(\rho_{R}, u_{R}, v_{R}, p_{R}\right)=(1,0,0,1 / \gamma): \text { Pre-shock }
\end{aligned}
$$

In his test, Quirk also introduced another parameter, i.e., grid perturbation $\varepsilon_{g}$ as $1 . e-3$ times the cell size. The perturbation was imposed to the centerline of a $2 \mathrm{D}$ duct through which a shock was propagating as:

$$
y_{\text {centerline }}= \begin{cases}y_{\text {original }}+\varepsilon_{g} & (\text { for } i: \text { odd }) \\ y_{\text {original }}-\varepsilon_{g} & (\text { for } i: \text { even })\end{cases}
$$

The shock can take all the possible locations inside the shock (in contrast to [3]), but may pass through those locations too quickly to be affected by every state. Roberts [8], on the other hand, chose a more slowly moving shock without grid perturbation $\left(\varepsilon_{g}=0\right)$. Thus, as reviewed here, moving shock anomalies have been studied by different researchers from different approaches.

\section{E. Present Setup for General Stationary and Moving Shocks}

The parameters involved in the present survey are summarized in Table 1. One can note that included are the choices in Quirk [1] of $\left(M, M_{s}\right)=(6,6)$ and those in Kitamura et al. [3] of $\left(M, M_{s}\right)=(6,0)$.

Depending on the magnitudes of $M$ and $M_{s}$, flow directions can change as shown in Fig. 7. The boundary conditions are such that Riemann invariants are conserved along characteristic curves and either inflow or outflow condition is satisfied according to the flow direction. Grid perturbation parameter $\varepsilon_{g}$ is set to be 1.e-3 times the cell size as default, and 0 and 1.e- 6 as alternatives. Flux functions are chosen from three categories $[15,20]$ in terms of robustness against shock and other properties: i) Roe scheme [17] is known to be vulnerable to carbuncle, even with Harten's entropy fix [21] [Roe (E-fix)]; ii) Van Leer's FVS [16], AUSMPW+ [29], RoeM2 [30], SLAU2 [26] are almost (but not perfectly) 
free from such anomalies; iii) $\mathrm{AUSM}^{+}$-up [18], LDFSS [28], and SLAU [19] are in the middle. The computations are run for 400 steps with $\mathrm{CFL}=0.4$ (Fig. 8). Following Quirk [1], the grid consists of $800 \times 20$ cells. The initial shock is placed at the interface between 25th and 26th cells $(\varepsilon=0.0)$.

\section{Numerical Results}

\section{A. Effects of Two Mach numbers $M_{s}$ and $M$}

Figure 9 summarizes Roe (E-fix) solutions for $M_{s}=0,0.5,1.0,1.5,3.0,6.0$, and $10.0\left(\varepsilon_{g}=1 . \mathrm{e}-3\right)$, according to the following criteria:

O: Stable. L1-norm of $v$-velocity residual remained the same order from the beginning.

$\Delta$ : Oscillatory, but no carbuncle. L1-norm of $v$-velocity residual grew over one order.

X: Carbuncle (total breakdown). The shock oscillated spatially over four cells or more in the freestream direction.

Here we used the L1-norm of velocity component $v$ (cross flow velocity component), which is supposed to be zero in a theoretical sense, as a measure of (multi-dimensional) shock anomalies. As seen, the stable and carbuncle solutions exist, but increase of $M_{s}$ allows higher $M$. Very fast moving shocks such as $\left(M, M_{s}\right)=(3,10)$ showed a stable solution, whereas a mildly fast moving shock $\left(M, M_{s}\right)=(3,2)$ was unstable. Typical visualized results are shown in Fig. 10 for $M=6 ; M_{\mathrm{s}}=0,1.5,6$ (corresponds to Quirk [1]), and 10. This figure also demonstrates that the faster the shock propagates (the higher the $M_{s}$ ), the more stable it is.

Figure 11 shows L1-norms of $v$-residuals, whose growth in time indicates multidimensional instability. As seen from Fig. 11a, the $M_{s}=0$ (stationary) shocks exhibit such unstable growth except for the $M=1.5$ (the weakest) case ( $M$ $<2$ : the weak shock); the second weakest $M=3$ shock shows slight delay of the growth of instability when $M_{s}=0.5<$ $0.44 M$ (slowly moving) in Fig. $11 \mathrm{~b}$; the two weakest shocks $M=1.5$ and 3 are stable for $M_{s}=6>0.44 M$ (fast moving) in Fig. 11c.

\section{B. Effects of Flux Functions}

Figure 12 shows the summarized results for AUSM+-up, SLAU, Van Leer, LDFSS, AUSMPW+, RoeM2, and SLAU2. Among them, AUSMPW+ and Van Leer's FVS are stable ${ }^{\dagger \dagger}$ except for the two cases of Van Leer in which $v$ appears but the shock itself looks symmetry (indicated by solid circles). SLAU2 and RoeM2 are almost always stable,

\footnotetext{
${ }^{\dagger \dagger}$ Nevertheless, this does not mean that they are always stable under any conditions. On meshes with high aspect ratio, AUSMPW+ maybe unstable [15].
} 
with only a few cases of slight $v$-velocity growth. This observation appears to contradict with [26] in which SLAU2 showed the best stability against shock anomalies while AUSMPW+ did not [3]. In [3] and [26], however, no grid perturbations were introduced. Thus, for perturbed grids, a multi-dimensional pressure weight function in AUSMPW+ may have acted favorably, whereas SLAU2 equipped with no such a term has been affected by the grid under particular setups. Furthermore, if the "Stable" criterion is relaxed to allow one order growth of $v$-velocity (i.e., two orders or more are called "Oscillations"), the solutions of SLAU2 will be all re-labelled as Stable.

$\mathrm{AUSM}^{+}$-up, SLAU, and LDFSS showed mixed solutions of Stable and Oscillatory. Nevertheless, all these are more robust than Roe (E-fix), and again, the faster the shock (the higher the $M_{s}$ ), the more stable the shock for AUSM ${ }^{+}-$ up (except for a stationary shock), for instance.

Figures 13-15 confirm that at $M=6$, all the solutions look stable for $\mathrm{AUSM}^{+}$-up, SLAU, and Van Leer (no oscillations are visible from these figures). However, showing these figures only may misguide the readers. After seeing the Fig. 12 together, we are aware of the fact that a different $\left(M, M_{s}\right)$ set yielded a different solution, and that we need such a broad picture to examine the performance of the flux functions. In other words, only one particular shock speed (e.g., the Quirk's test [1]) may not be enough to support the robustness of the flux function against shocks.

In fact, the stationary shocks $\left(M_{s}=0\right)$ behaved totally differently from the moving ones (Figs. $\left.11 \mathrm{a}, 11 \mathrm{~b}\right)$. This is understandable considering the fact that each flux function has its own "comfortable" shock location relative to grid lines [3]. In $\mathrm{AUSM}^{+}$-up cases, for instance, the stationary shocks located exactly at the cell interface showed no sign of shock anomalies (Figs. 12a and 13a).

However, on the contrary to the $\mathrm{AUSM}^{+}$-up, the stationary shocks are less stable than the moving counterparts in SLAU (Fig. 12b): In very general, SLAU showed more stable moving shocks compared to AUSM ${ }^{+}$-up, but this is opposite for stationary shocks at cell interface. Furthermore, since there are many stable moving shocks in Fig.12b, it is hard to find any tendency according to $\left(M, M_{s}\right)$ combinations, for this particular flux function.

The final flux function focused on is Van Leer's FVS. This flux is known to be very robust against the (stationary) shock anomalies, and this still holds for moving shocks according to Figs.12c and 15 . Thus, the stable combinations of $\left(M, M_{s}\right)$ and the overall robustness against the shock differ from one flux function to another. Nevertheless, the $0.44 M$ (approximate signal speed) is useful to categorize the results for simple estimations as follows: 
- [Group I (Stationary Shock: $M_{s}=0, M \geq 2$ )] Since the shock did not cross any cells, there should be no influence from the grid perturbation. Each flux function has its own "comfortable" shock location relative to grid lines [3], and $\mathrm{AUSM}^{+}$-up, LDFSS, and AUSMPW+ showed no instability regardless of shock intensity, $M$.

- [Group II (Slowly Moving Shock: $0<M_{s}<0.44 M, M \geq 2$ )] Since the shock crosses a perturbed grid with a long time (during which the instabilities can grow) and since numerical noises from the left (downstream) region (Fig. 7a) can affect the shock itself (as explained in Sec. III-B), the solution is most likely to exhibit carbuncle. In Roe (E-fix), for instance, the solutions are usually carbuncle with a few exceptions of slight oscillation cases.

- [Group III (Fast Moving Shock: $0.44 M<M_{s}, M \geq 2$ )] Since the shock crosses a perturbed grid with a short time and numerical noise from the left (downstream) region hardly reaches the shock itself, the solution is less likely to exhibit anomalies than Group II. The solutions are mixed with stable, oscillatory, and carbuncle cases. Although the border between the Groups II and III is not clear (since it is based on $0.44 M$, approximated signal speed), the Group III tends to be more stable than the Group II (except for SLAU).

- [Group IV (Weak Shock: $M<2)]$ The shock intensity is not strong enough to provoke carbuncle. The solutions are usually free from such anomalies.

The reason why SLAU behaved differently would be related to its inherent shock-capturing feature [20]. Roe and $\mathrm{AUSM}^{+}$-up captures a shock sharply, while SLAU (as well as SLAU2 and Van Leer) represents it with a few cells. Thus, SLAU is less sensitive to the moving shock speed, since it already contains the (numerical) shock internal structure (Fig. 16a), as opposed to Roe or $\mathrm{AUSM}^{+}$-up (Fig. 16b). AUSMPW+ and RoeM2, having a multi-dissipation term to successfully stabilize the shock, are also insensitive to the shock propagation speed.

\section{Effects of Grid Perturbation $\varepsilon_{g}$}

Finally, Fig. 17 shows the effects of the grid perturbation $\varepsilon_{g}$, which is reduced from the default value of $1 . e-3$ to 1.e-6, and even 0. As seen, the solutions (common to both $\varepsilon_{g}=1 . e-6$ and 0 ) look more stable: Only the unstable solutions are found at $\left(M, M_{s}\right)=(10,0)$ and $(6,0)$, i.e., stationary shocks; otherwise, the velocity $v$ is very small (within 1.e-12). Its growth implies potential carbuncle at a very later stage (i.e., after very long computation)

Nevertheless, within the present framework, neither of $\varepsilon_{g}=1 . e-6$ nor 0 showed a clear sign of carbuncle, and no distinct difference was observed between them. Thus, the grid perturbation $\varepsilon_{g}$ has minor effect on the results as long as it is taken to be small enough. On the other hand, in Quirk [1], $\varepsilon_{g}=1 . e-3$ was chosen which was large enough to 
trigger the carbuncle and other shock anomalies at an early stage of computations. Thus, $\varepsilon_{g}=1 . \mathrm{e}-3$ is found to be a good value to check the occurrence of shock anomalies in a realistic time.

\section{Conclusions}

As a systematic and thorough benchmark, we carried out numerical experiments for a variety of shock intensity Mach number $M$, and shock propagation Mach number $M_{s}$, with different flux functions and different levels of grid perturbations. According to the difference between the flux functions,

- SLAU, Van Leer, AUSMPW+, RoeM2, SLAU2: They are not affected by shock propagation speeds, since the shock is captured over a few cells and/or a multi-dimensional dissipation term successfully stabilizes the shock.

- Roe, AUSM+-up, LDFSS: They are easily affected by shock propagation speeds, since they tend to capture the shock sharply (within at most one cell).

Then, for the latter fluxes, the numerically captured shock waves are categorized into the four groups having different degrees of robustness against shock anomalies according to $M$ and $M_{s}$,:

I. Stationary shocks $\left(M_{s}=0 ; M \geq 2\right)$ : Either more or less stable than moving ones, depending on the flux functions.

II. Slowly moving shocks $\left(0<M_{s} \leq 0.44 M ; M \geq 2\right)$ : This is the most severe case, since they are affected by both grid perturbation and numerical noises from the left (downstream) region.

III. Fast moving shocks $\left(0.44 M<M_{s} ; M \geq 2\right)$ : Less likely to exhibit shock anomalies, since they are less influenced by the left (downstream) region.

IV. Weak $(M<2)$ shocks: Usually stable.

Thus, only a particular set of $\left(M, M_{s}\right)$ [which is only a fraction of the broader $\left(M, M_{s}\right)$ charts presented herein] cannot support stability of a flux function enough. Finally, the present default choice of grid perturbation following Quirk (1.e-3 the cell size) turned out to be big enough to trigger the shock anomalies at earlier stages.

Now the ultimate goal has been set: We will explore the universal flux function which is always robust against shock anomalies for all the $\left(M, M_{s}\right)$, while applicable to a contact discontinuity capturing, hypersonic wall heating, and all-speed flow computations. Such a study is left as a future work, but SLAU2 used with the minmod slope limiter is recommended, for the time being [20]. 


\section{Acknowledgments}

This work was partially conducted while Keiichi Kitamura was under supervision of Eiji Shima at JAXA, supported by JSPS (Japan Society for the Promotion of Science). Tsutomu Saito, Muroran Institute of Technology, Japan, gave us a helpful comment. We thank their cooperation.

\section{References}

[1] Quirk, J. J., “A Contribution to the Great Riemann Solver Debate,” International Journal for Numerical Methods in Fluids, Vol. 18, No. 6, 1994, pp. 555-574. doi:10.1002/fld.1650180603

[2] Pandolfi, M. and D’Ambrosio, D., "Numerical Instabilities in Upwind Methods: Analysis and Cures for the "Carbuncle" Phenomenon,” Journal of Computational Physics, Vol. 166, No. 2, 2001, pp.271-301. doi:10.1006/jcph.2000.6652

[3] Kitamura, K., Roe, P., and Ismail, F., "Evaluation of Euler Fluxes for Hypersonic Flow Computations,” AIAA Journal, Vol. 47, 2009, pp.44-53. doi: 10.2514/1.33735

[4] Zaide, D. and Roe, P.L., "Shock Capturing Anomalies and the Jump Conditions in One Dimension,” AIAA Paper 20113686, 2011.

[5] Barth, T. J., “Some Notes on Shock-Resolving Flux Functions Part 1: Stationary Characteristics,” NASA TM-101087, 1989.

[6] Chauvat, Y., Moschetta, J.M., and Gressier, J., "Shock Wave Numerical Structure and the Carbuncle Phenomenon," International Journal for Numerical Methods in Fluids, Vol. 47, 2005, pp. 903-909. doi:10.1002/fld.916

[7] Roe, P.L., "Fluctuations and Signals-A Framework for Numerical Evolution Problems," Numerical Methods for Fluid Dynamics, edited by K. W. Morton, and M. J. Baines, Academic Press, New York, 1982, pp. 219-257.

[8] Roberts, T. W., “The Behavior of Flux Difference Splitting Schemes Near Slowly Moving Shock Waves,” Journal of Computational Physics, Vol. 90, No. 1, 1990, pp. 141-160. doi:10.1016/0021-9991(90)90200-K

[9] Johnsen, E., “Analysis of Numerical Errors Generated by Slowly Moving Shock Waves,” AIAA Journal, Vol. 51, No.5, 2013, pp.1269-1274. doi: 10.2514/1.J051884

[10] Jin, S., and Liu, J. G., “The Effects of Numerical Viscosities: 1. Slowly Moving Shocks,” Journal of Computational Physics, Vol. 126, No. 2, 1996, pp. 373-389. doi:10.1006/jcph.1996.0144

[11] Karni, S., and Canic, S., "Computations of Slowly Moving Shocks," Journal of Computational Physics, Vol. 136, No. 1, 1997, pp. 132-139. doi:10.1006/jcph.1997.5751

[12] Arora, M., and Roe, P. L., “On Postshock Oscillations Due to Shock Capturing Schemes in Unsteady Flows,” Journal of Computational Physics, Vol. 130, No. 1, 1997, pp. 25-40. doi:10.1006/jcph.1996.5534

[13] Dumbser, M., Moschetta, J. M., and Gressier, J., “A Matrix Stability Analysis of the Carbuncle Phenomenon,” Journal of Computational Physics, Vol. 197, No. 2, 2004, pp. 647-670. doi:10.1016/j.jcp.2003.12.013 
[14] Sanders, R., Morano, E., and Druguetz, M. C., "Multidimensional Dissipation for Upwind Schemes: Stability and Applications to Gas Dynamics,” Journal of Computational Physics, Vol. 145, No. 2, 1998, pp. 511-537. doi:10.1006/jcph.1998.6047

[15] Kitamura, K., Shima, E., Nakamura, Y., and Roe, P., "Evaluation of Euler Fluxes for Hypersonic Heating Computations," AIAA Journal, Vol.48, 2010, pp.763-776. doi:10.2514/1.41605.

[16] van Leer, B., "Flux Vector Splitting for the Euler Equations," Eighth International Conference of Numerical Methods in Fluid Dynamics, Lecture Notes in Physics, Vol. 170, Springer, Berlin, 1982, pp. 507-512.

[17] Roe, P.L., “Approximate Riemann Solvers, Parameter Vectors, and Difference Schemes,” Journal of Computational Physics, Vol. 43, 1981, pp.357-372. doi:10.1016/0021-9991(81)90128-5

[18] Liou, M. S., “A Sequel to AUSM, Part II: AUSM+-up for All Speeds,” Journal of Computational Physics, Vol. 214, No. 1, 2006, pp. 137-170. doi:10.1016/j.jcp.2005.09.020

[19] Shima, E. and Kitamura, K., "Parameter-Free Simple Low-Dissipation AUSM-Family Scheme for All Speeds," AIAA Journal, Vol.49, No.8, 2011, pp.1693-1709. doi:10.2514/1.55308

[20] Kitamura, K., “Assessment of SLAU2 and Other Flux Functions with Slope Limiters in Hypersonic Shock-Interaction Heating," Computers \& Fluids, Vol.129, 2016, pp.134-145. doi:10.1016/j.compfluid.2016.02.006

[21] Harten, A., "High Resolution Schemes for Hyperbolic Conservation Laws," Journal of Computational Physics, Vol. 49 (1983), pp.357-393. doi:10.1016/0021-9991(83)90136-5

[22] Godunov, S. K., “A Finite Difference Method for the Numerical Computation of Discontinuous Solutions of the Equations of Fluid Dynamics,” Matematicheskii Sbornik/Izdavaemyi Moskovskim Matematicheskim Obshchestvom, Vol. 47, No. 3, 1959, pp. 271-306.

[23] Toro E.F., Spruce M., and Speares W., "Restoration of the contact surface in the HLL Riemann solver," Shock Waves, 1994, Vol. 4, pp.25-34.

[24] Hänel, D, Schwane, R., and Seider, G., "On the Accuracy of Upwind Schemes for the Solution of the Navier-Stokes Equations," AIAA Paper 87-1105, 1987.

[25] Einfeldt, B., “On Godunov-Type Methods for Gas Dynamics,” SIAM Journal on Numerical Analysis, Vol. 25, No.2, 1998, pp.294-318. doi:10.1137/0725021

[26] Kitamura, K. and Shima, E., “Towards shock-stable and accurate hypersonic heating computations: A new pressure flux for AUSM-family schemes,” Journal of Computational Physics, Vol.245, 2013, pp.62-83. doi:10.1016/j.jcp.2013.02.046

[27] Liou, M.-S., “A Sequel to AUSM: AUSM+,” Journal of Computational Physics, Vol. 129 (1996), pp.364-382.

[28] Edwards, J.R., “Towards Unified CFD Simulation of Real Fluid Flows,” AIAA Paper 2001-2524, 2001.

[29] Kim, K. H., Kim, C., and Rho, O. H., "Methods for the accurate computations of hypersonic flows: I. AUSMPW+ scheme." 
Journal of Computational Physics, 174.1 (2001): 38-80.

[30] Kim, S., et al., "Cures for the shock instability: Development of a shock-stable Roe scheme.” Journal of Computational Physics, 185.2 (2003): 342-374.

[31] Men'shov, I.S., and Nakamura, Y., "Numerical Simulations and Experimental Comparisons for High-Speed Nonequilibrium Air Flows,” Fluid Dynamics Research, Vol. 27 (2000), pp.305-334.

[32] Anderson, J. D., Jr., Modern Compressible Flow with Historical Perspective, 3rd ed., McGraw-Hill, New York, 2003.

[33] Horvath, T.J., "Experimental Aerothermodynamics in support of the Columbia Accident Investigation,” AIAA 2004-1387, 42nd AIAA Aerospace Sciences Meeting and Exhibit 5 - 8 January 2004, Reno, Nevada. 


\section{Figures}

a)

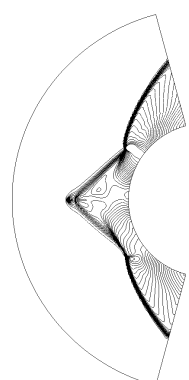

b)

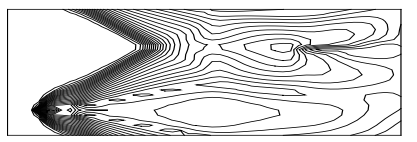

Fig. 1 Carbuncle solutions, a) ahead of blunt-body and b) in computational domain composed of squares.

a)
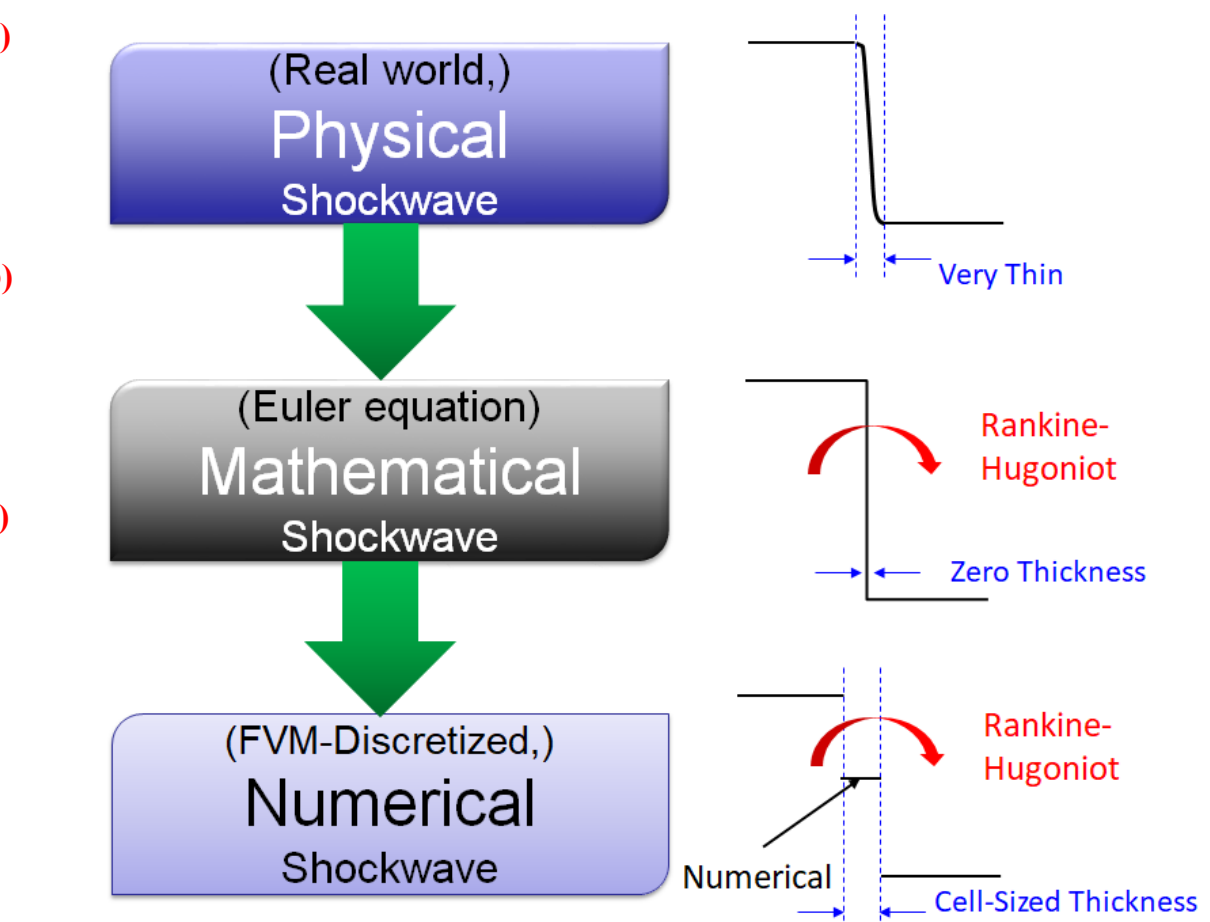

Fig.2 Schematics of physical, mathematical, and numerically-expressed shockwaves.

a)

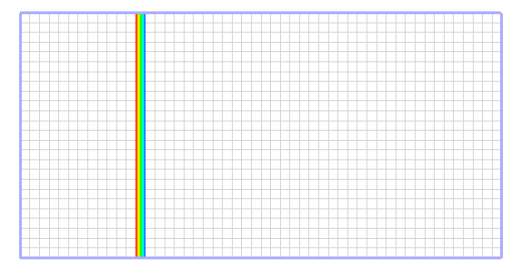

b)

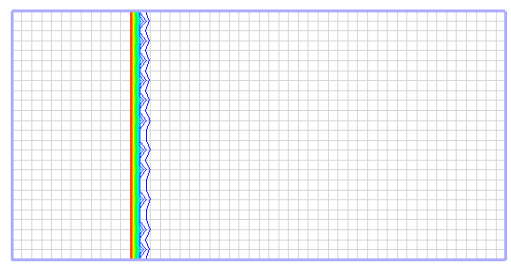

Fig. 3 a) $\varepsilon=0.0$ (Stable) and b) $\varepsilon=0.5$ (Oscillatory) results using $\operatorname{AUSM}^{+}$-up $\left(M_{\mathrm{s}}=0 ; M=6\right)$. 
a)

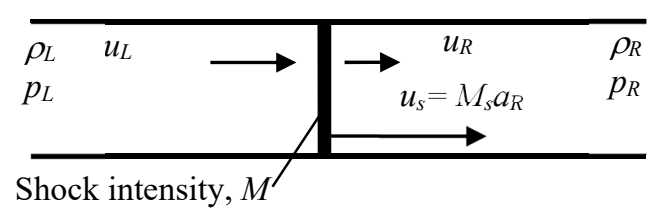

b)

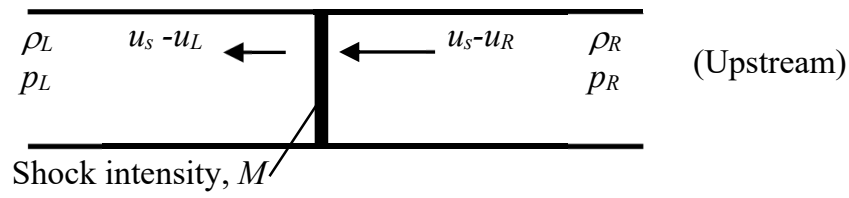

Fig. 4 Schematic of pre- and post-shock relations: a) Laboratory coordinate system, and b) Shock coordinate system.

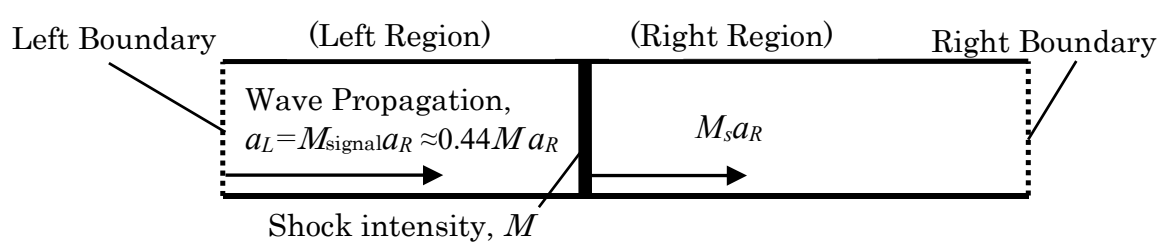

Fig. 5 Schematic of wave propagation, and left and right regions (Laboratory coordinate system).

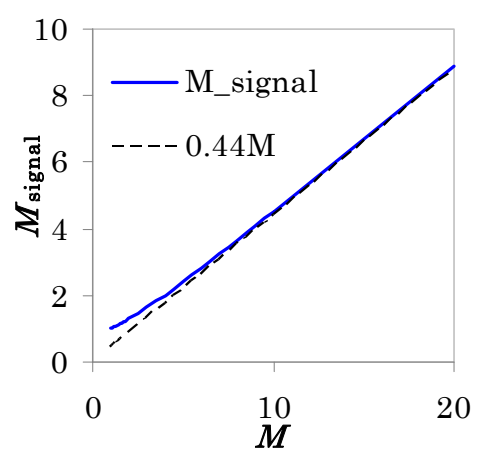

Fig. $6 M_{\text {signal }}$ versus $M$ 
a)

BC: Riemann Invariants

$\mathrm{BC}$ : Riemann Invariants

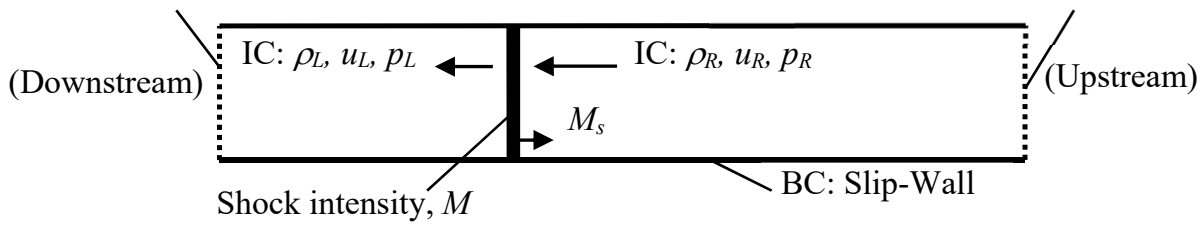

b)

BC: Riemann Invariants

BC: Riemann Invariants

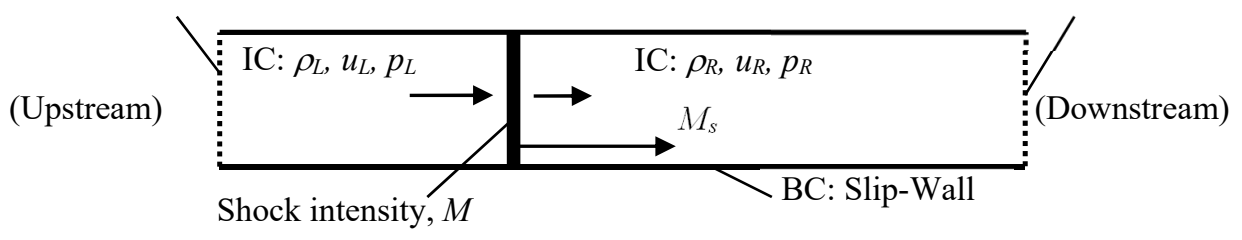

Fig. 7 Typical initial (IC) and boundary (BC) conditions for a) flows of right to left and b) flows of left to right moving shock cases (Laboratory coordinate system).

a)

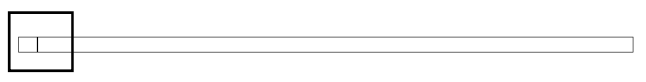

b)

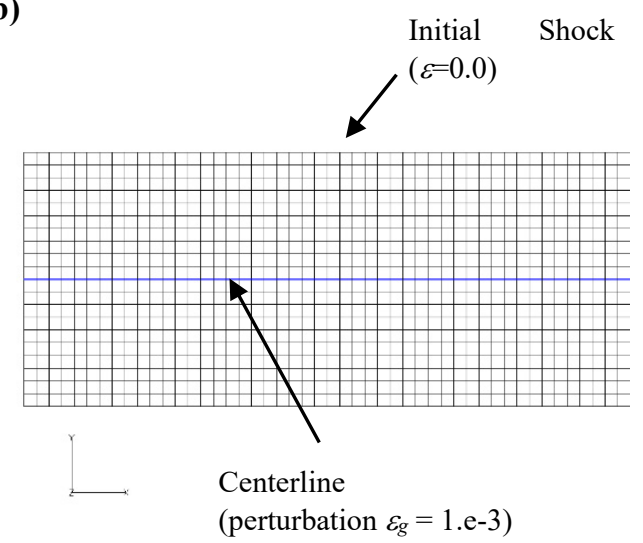

Fig. 8 Moving shock test grid ( $\left.\varepsilon_{\mathrm{g}}=1 . \mathrm{e}-3\right)$, a) overview and b) close-up view. 


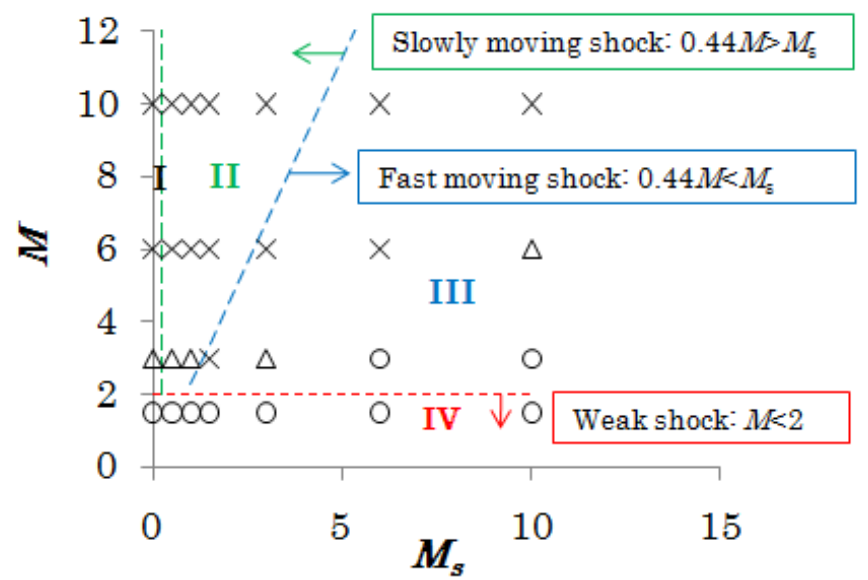

Fig. 9 Moving shock test results $\left(\varepsilon_{\mathrm{g}}=1 . \mathrm{e}-3\right)$, Roe (E-fix). O: Stable. L1-norm of v-velocity residual remained the same order from the beginning; $\Delta$ : Oscillatory, but no carbuncle. L1-norm of $v-$ velocity residual grew over one order; $\times$ : Carbuncle (total breakdown).

a)

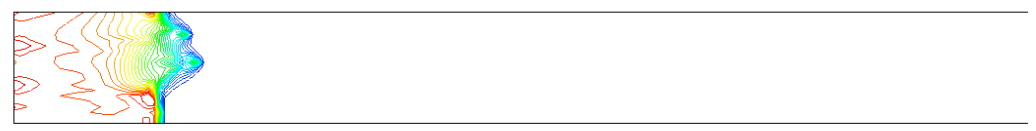

b)

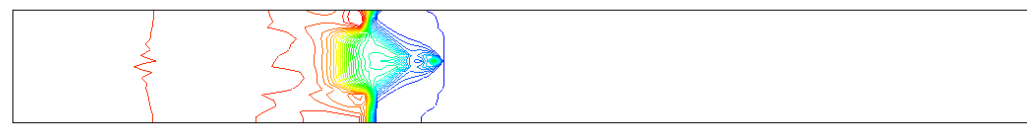

c)

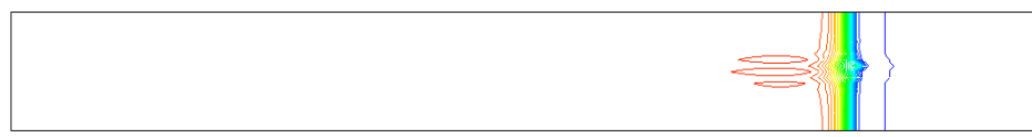

d)

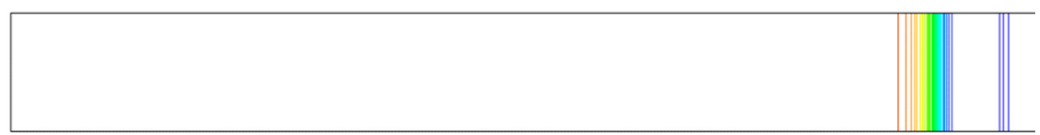

Fig. 10 Moving shock test, $M=6$ (Roe (E-fix), $\varepsilon_{\mathrm{g}}=1 . \mathrm{e}-3,400$ steps); a) $M_{\mathrm{s}}=0$, b) $M_{\mathrm{s}}=1.5$, c) $M_{\mathrm{s}}=6$, and d) $M_{\mathrm{s}}=10$. The faster the shock, the more stable it tends to be. 
a)

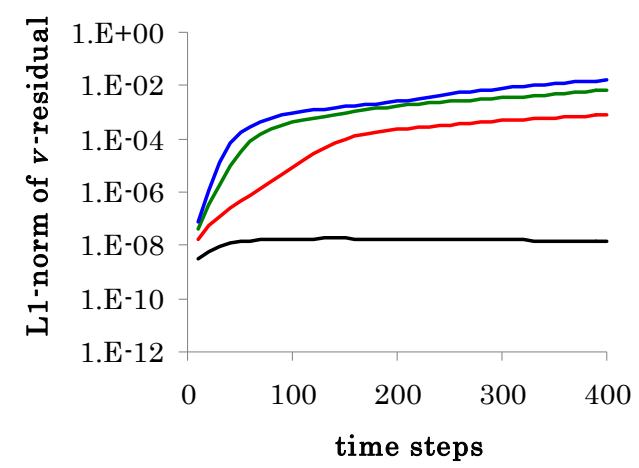

b)

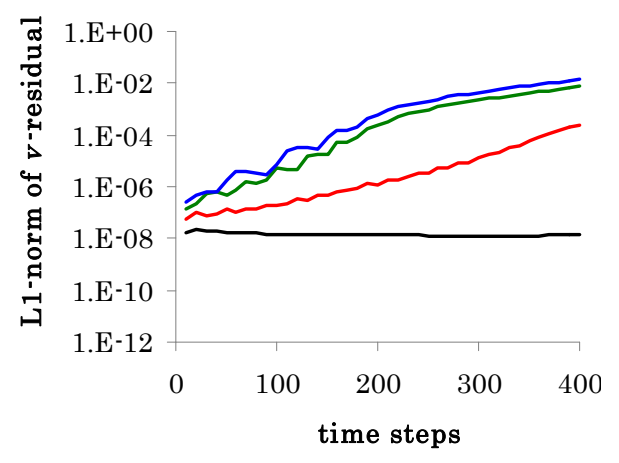

c)

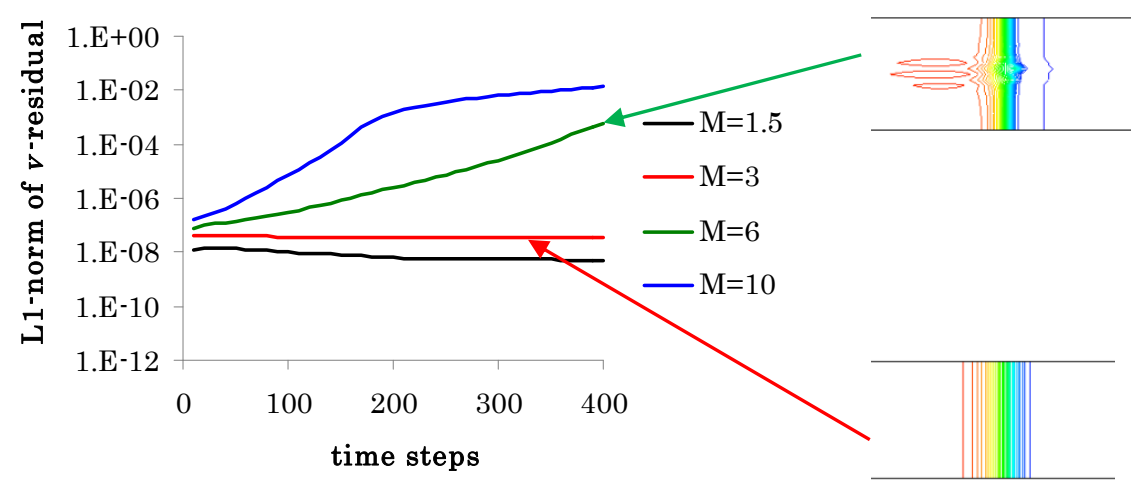

Fig. 11 L1-norm histories of $v$-velocity residual for moving shock test: $M=1.5,3,6$, and 10 (Roe (E-fix), $\varepsilon_{\mathrm{g}}=1$.e3, 400 steps), a) $M_{s}=0.0$ (stationary), b) $M_{s}=0.5$ (slowly moving), and c) $M_{s}=6$ (fast moving). 
a)

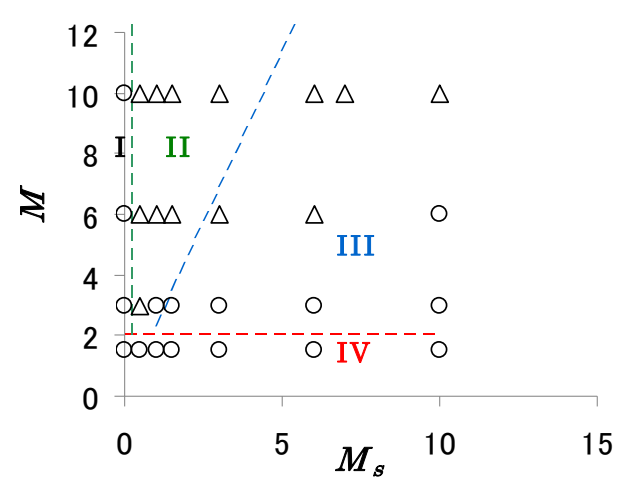

c)

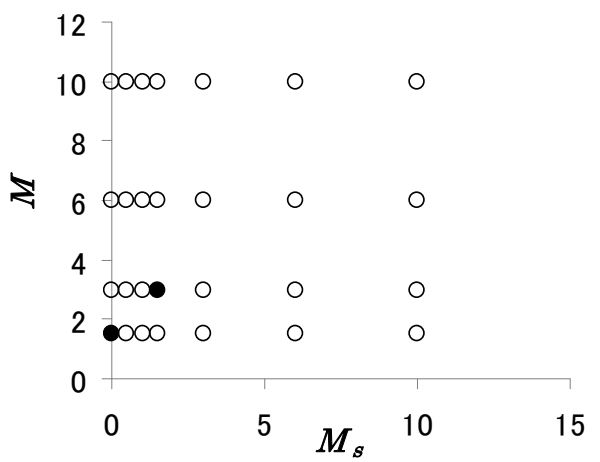

e)

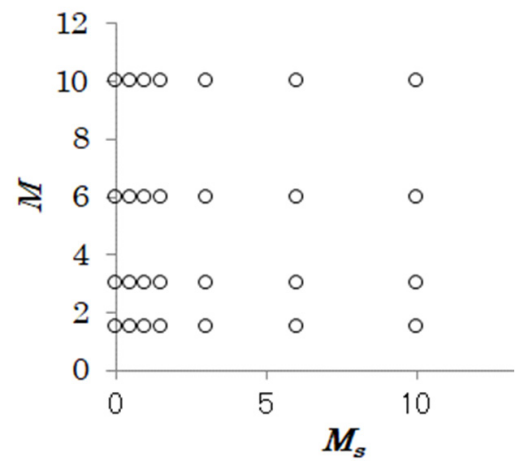

b)

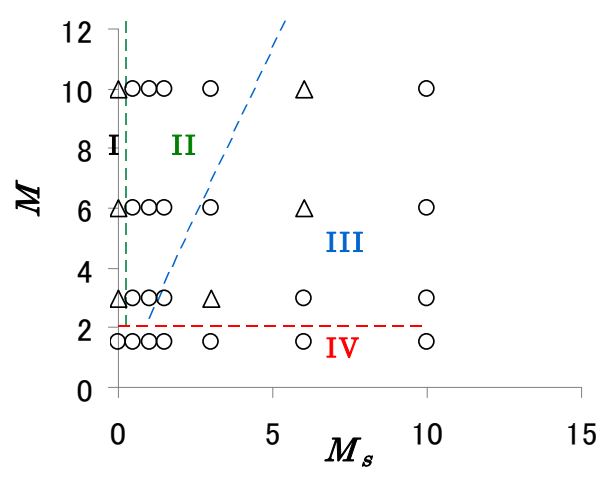

d)

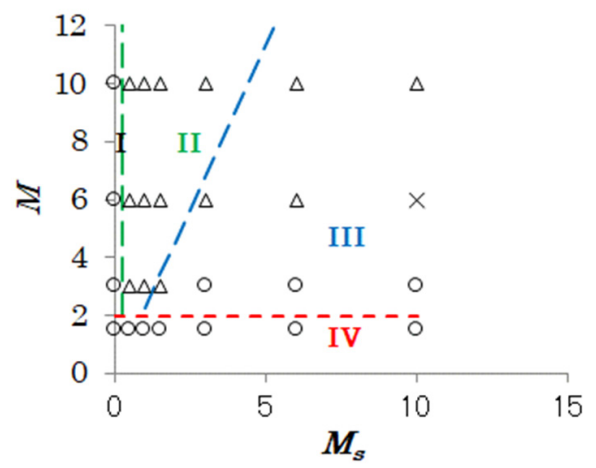

g)

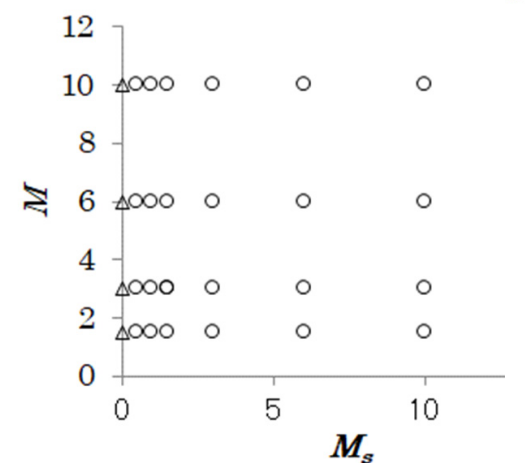

Fig. 12 Moving shock test results ( $\left.\varepsilon_{\mathrm{g}}=1 . \mathrm{e}-3\right)$ : a) AUSM+-up, b) SLAU, c) Van Leer, d) LDFSS, e) AUSMPW+, f) RoeM2, and g) SLAU2. O: Stable. L1-norm of $v$-velocity residual remained the same order from the beginning; $\Delta$ : Oscillatory, but no carbuncle. L1-norm of $v$-velocity residual grew over one order; $\times$ : Divergence [Carbuncle (total breakdown) did not occur for those fluxes]; $\bullet$ : Shock itself is stable, but $v$ appeared at the boundary. 
a)

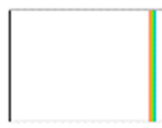

b)

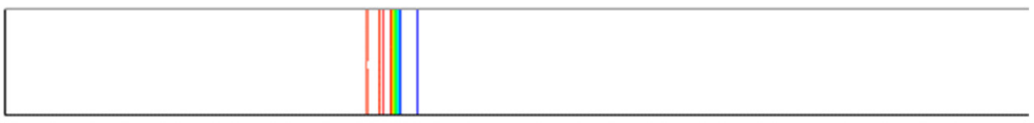

c)

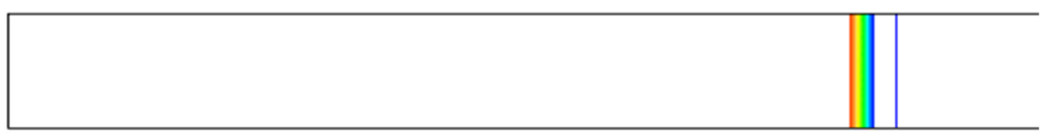

d)

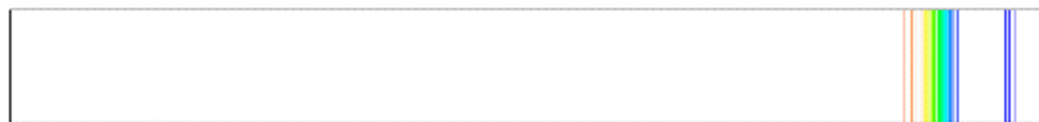

Fig. 13 Moving shock test, $M=6\left(\mathrm{AUSM}^{+}\right.$-up, $\varepsilon_{\mathrm{g}}=1 . \mathrm{e}-3,400$ steps $)$; a) $M_{\mathrm{s}}=0$, b) $M_{\mathrm{s}}=1.5$, c) $M_{\mathrm{s}}=6$, and d) $M_{\mathrm{s}}=10$. The faster the shock, the more stable it tends to be.

a)

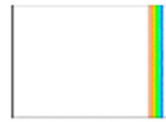

b)

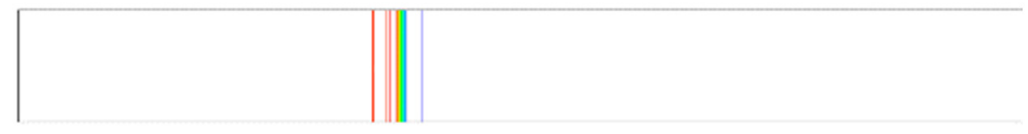

c)

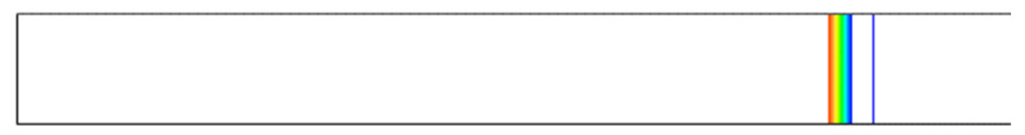

d)

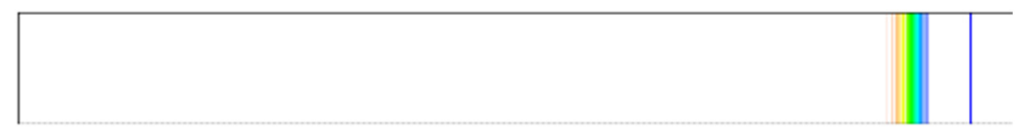

Fig. 14 Moving shock test, $M=6$ (SLAU, $\varepsilon_{\mathrm{g}}=1 . \mathrm{e}-3,400$ steps); a) $M_{\mathrm{s}}=0$, b) $\left.M_{\mathrm{s}}=1.5, \mathrm{c}\right) M_{\mathrm{s}}=6$, and d) $M_{\mathrm{s}}=10$. 
a)

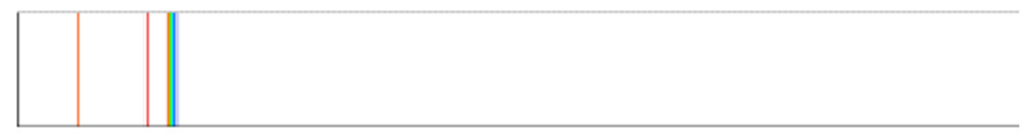

b)

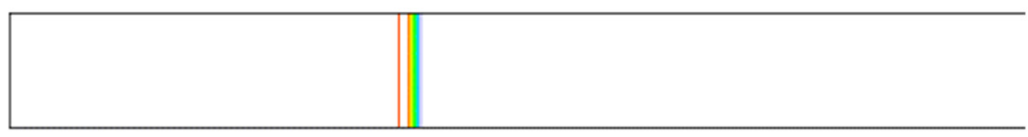

c)

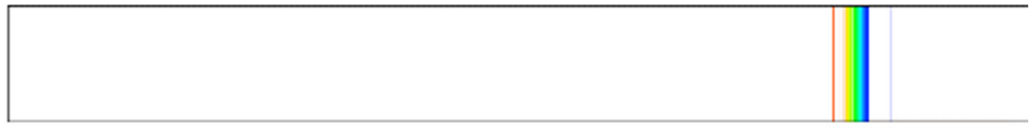

d)

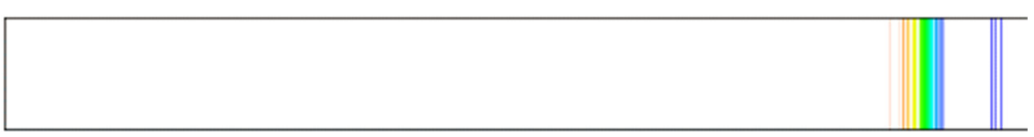

Fig. 15 Moving shock test, $M=6$ (Van Leer, $\varepsilon_{\mathrm{g}}=1 . \mathrm{e}-3,400$ steps); a) $M_{\mathrm{s}}=0$, b) $M_{\mathrm{s}}=1.5$, c) $M_{\mathrm{s}}=6$, and d) $M_{\mathrm{s}}=10$.

a)

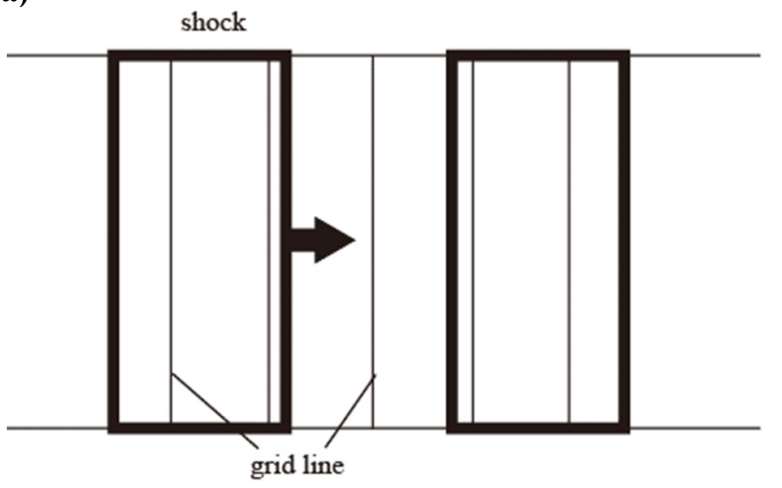

b)

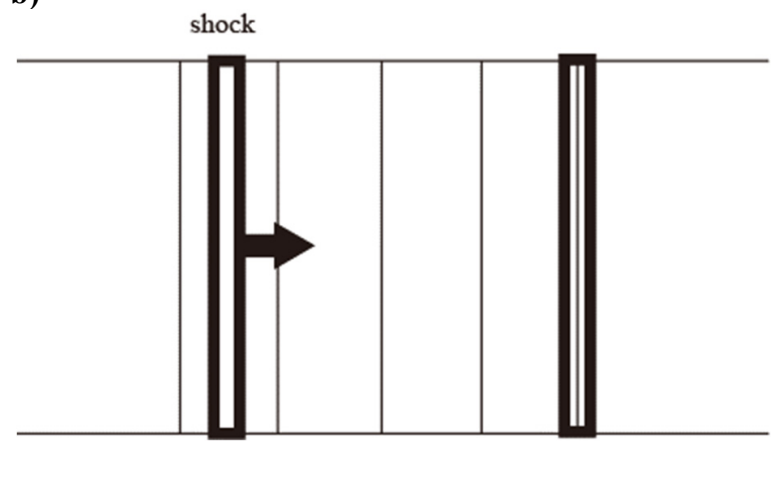

Fig. 16 Schematics of moving shocks captured by a) SLAU and b) Roe.

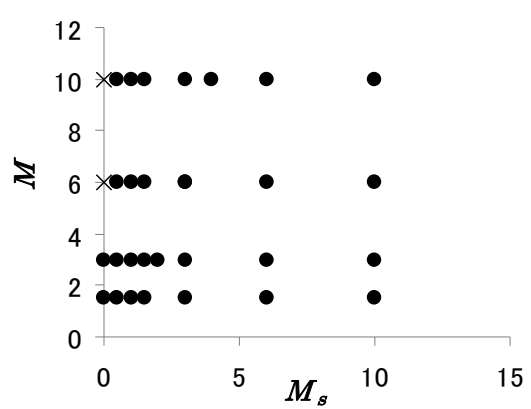

Fig. 17 Moving shock test results (Roe (E-fix), $\varepsilon \mathrm{g}=1 . e-6$ or 0). $\times$ : Carbuncle (total breakdown); •: rapid growth of $v$, though still very small value $\left(O\left(10^{-12}\right)\right.$ or smaller). 
Table

Table 1. Parameters in the present survey

Shock propagation Mach number $M_{\mathrm{s}}$ $0,0.5,1,1.5,3,6,10$

Shock intensity Mach number $M$ $1.5,3,6,10$

Grid perturbation $\varepsilon_{\mathrm{g}}$ $0,1 . e-6,1 . e-3$

Flux functions

Roe (E-fix), Van Leer, $\mathrm{AUSM}^{+}$-up, SLAU,

LDFSS, AUSMPW+, RoeM2, SLAU2 